\title{
Double Femtosecond Laser Created Incomplete Flaps After Repeated Docking
}

\author{
Amr Mounir ${ }^{1 *}$, Engy Mohamed Mostafa ${ }^{2}$, Mohamed Gamal Elghobaier ${ }^{3}$ and Osama Ali Mohamed ${ }^{4}$ \\ ${ }^{1}$ Department of Ophthalmology, Sohag Faculty of Medicine, Egypt \\ ${ }^{2}$ Department of Ophthalmology, Sohag Faculty of Medicine, Egypt \\ ${ }^{3}$ Egyptian police hospitals, Egypt \\ ${ }^{4}$ Department of Ophthalmology, Sohag Faculty of Medicine, Egypt
}

*Corresponding author: Amr Mounir, Department of Ophthalmology, Sohag

Received Date: March 04, 2019

Faculty of Medicine, Egypt.

\begin{abstract}
Purpose: To report a case of femtosecond laser flap complication in the form two incomplete flaps after repeated separate docking procedures.

Methods: a 28-year-old female complaining of bilateral defective vision due to high myopia in right eye and subjected to right Femtosecond laser for flap creation for laser-assisted in situ keratomileusis (Lasik). After suction release we found that the created flap was decentered with flap edge near the pupil which will interfere with excimer laser ablation. So, the decision was to postpone the surgery. Another trial for Femtosecond laser flap creation with the same parameters of the previous trial with the same flap depth $90 \mathrm{um}$. During flap lifting the flap was found to be divided into 2 parts, one peripheral crescent the other is incomplete circular flap. Both parts were lifted with underlying wide ablation zone. After excimer laser treatment, both parts were repositioned to their respective places. Contact lens was applied at the end of the procedure.
\end{abstract}

Result: After 2 months of follow up complete healing of the flap has occurred with satisfactory refractive result.

Conclusion: Flap decentration and incomplete flaps can occur with femtosecond laser flap creation. More notable conclusion is that femto flap edge healing is not so tight as thought before.

Keywords: Femtosecond laser; Docking; Incomplete flaps

\section{Introduction}

Femtosecond laser-assisted in situ keratomileusis (FemtoLASIK) is the latest successful innovation in refractive surgery because of its superior outcome, long-term stable results, and reduced complications compared to other procedures [1]. The first phase of LASIK procedure, the creation of a corneal flap, is the most critical step of LASIK and it affects the visual outcome of the whole procedure [2]. Conventional mechanical microkeratome was used to make flaps that were thinner in the center compared to periphery which could lead to buttonhole perforations. This flap-related complication has been dramatically overcome by femtosecond laser that creates corneal flaps with uniform thickness.

In this case report study, we report a case of flap complication of Femtosecond laser created flaps.

\section{Case report}

A 28 years old lady complaining of bilateral defective vision due to high myopia seeking refractive surgery. The uncorrected visual acuity (UCVA) in the right eye (RE) was 1.2 Log MAR corrected to 0.18 by a refraction of -7.50Ds -1.00Dc x12, while the UCVA in the left eye (LE) was $1.2_{\text {Log }}$ MAR corrected to 0.3 by a refraction of -6.50 Ds -1.50Dc x196. Corneal topography was done for both eyes by Sirius Scheimpflug Analyzer (CSO, Florence, Italy) and the patient was diagnosed as normal and thinnest pachymetry was $530 \mu \mathrm{m}$ in Right eye and $535 \mu \mathrm{m}$ in Left eye with normal topographical map and no risk factors in both eyes.

After counseling with the patient, the decision was to do bilateral Femtosecond laser for thin $(90 \mu \mathrm{m})$ flap creation We 
calculated the possibility of correcting the whole refractive error taking into account that the residual stromal bed would be $300 \mu \mathrm{m}$. We also chose creating the flap with femtolaser to allow a regular ultrathin flap $(90 \mu \mathrm{m})$ leaving more stromal bed for the correction. As each diopter needs $13 \mu \mathrm{m}$ of the bed. This would allow us to correct in RE and in LE.

Before the surgery, topical anesthetic 0.4\% Benoxinate hydrochloride was instilled into the operated eyes. The corneal flap was cut with femtosecond laser by IntraLase (iFS, Abbott) the aimed flap thickness was $90 \mu \mathrm{m}$ in both eyes. Femtosecond laser parameters for the corneal flap creation included bed energy level of $0.85 \mu \mathrm{J}$, a side-cut energy level of $0.95 \mu \mathrm{J}$ with superior hinge orientation, flap diameter $9.00 \mathrm{~mm}$, side cut angle 90 degree and hinge angle 90 degree. In the right eye, docking procedure started by placing the suction ring between the eyelids, the suction ring was positioned over the cornea, relatively concentric to the limbus, and then vacuum was initiated. Integrated joystick was used for docking with the interface's disposable lens. Femtosecond Laser had been initiated to create the flap.

After suction release we found that the created flap was decentered with flap edge near the pupil edge which will interfere with excimer laser ablation (6mm ablation zone) safety. If we had proceeded with ablation it would have compromised the nearby epithelium concentric to the decentered flap leading to severe treatment decentration and would have affected healing and increased the incidence of epithelial ingrowth. So, the decision was to postpone the surgery and not try to dissect the flap from the bed to allow good flap healing and a therapeutic contact lens was applied for 24 hours.

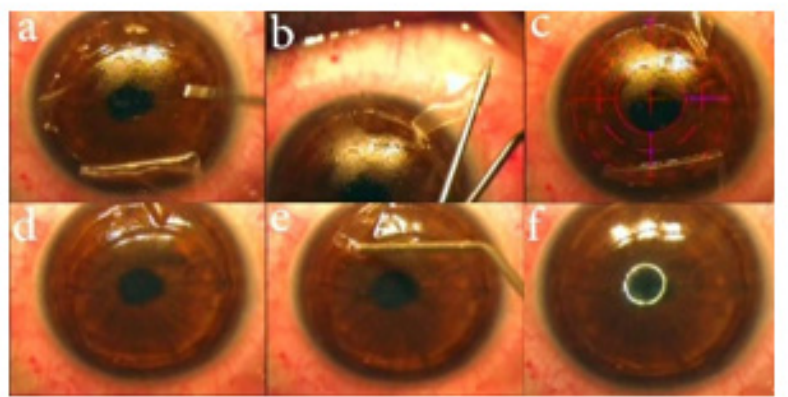

Figure 1: a) incomplete circular flap lift b) crescentic flap lift c) Excimer laser ablation d, e) flap repositioning f) complete flap alignment with regular illumination ring.

We followed up the patient for 3 months to ensure complete flap healing which is expected with femtosecond laser a second trial for Femtosecond laser flap creation was decided in the same parameters of the previous trial with the same flap depth 90 um to save more residual stromal bed. We were convinced that complete flap edge healing has occurred by after this duration especially we didn't try to lift the flap. This time we made sure that the cornea was totally centralized before Femtosecond Laser had been initiated. The second created flap was central with its edge away from the pupil to the extent that allow excimer laser ablation on lifting the flap, we found it to be divided into 2 parts, one peripheral crescent the other is incomplete circular flap. (Figure 1a, b) Both parts of the flap were attached to the superior hinge at a point. Going through with ablation this time as the flap was centered other parts were lifted meticulously. The excimer laser treatment was started immediately thereafter using the VISX S4IR. Both parts had been repositioned to their respective place with contact therapeutic contact lens application with regular ring of illumination. (Figure 1c, d, e, f). The second eye was treated in the second session by femtosecond laser with excimer laser ablation with same parameters and passed uneventful (Figure 1).

Postoperative medications included topical antibiotics eye drops ( $0.3 \%$ Gatifloxacin 5 times/day for one week), Topical steroid eye drops (1\% Prednisolone acetate 5 times/day for one week), Lubricant eye drops and systemic non-steroidal anti-inflammatory drugs.

The patient's follow up was for 2 months. Complete healing of the flap has occurred (Figure 2) with normal postoperative Corneal topography (Figure 3). The uncorrected visual acuity in the right eye was $0.3_{\text {Log }}$ MAR (satisfactory to the patient especially in near vision) corrected to 0.18 by a residual refraction of $-0.75 \mathrm{Ds}-0.50 \mathrm{Dc}$ axis 105 . The corneal surface was regular as well. The uncorrected visual acuity in the left eye was 0.3 with refraction of $-0.50 \mathrm{Ds}$ -0.50Dc axis 105 .

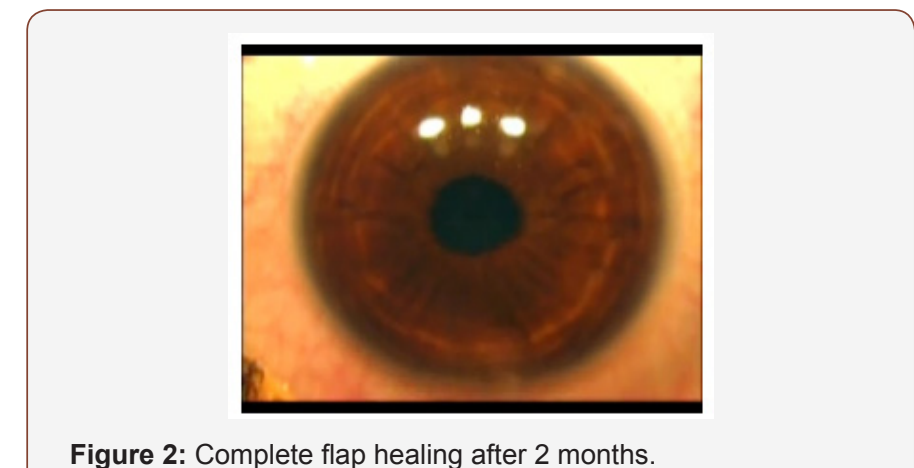

Figure 2: Complete flap healing after 2 months.

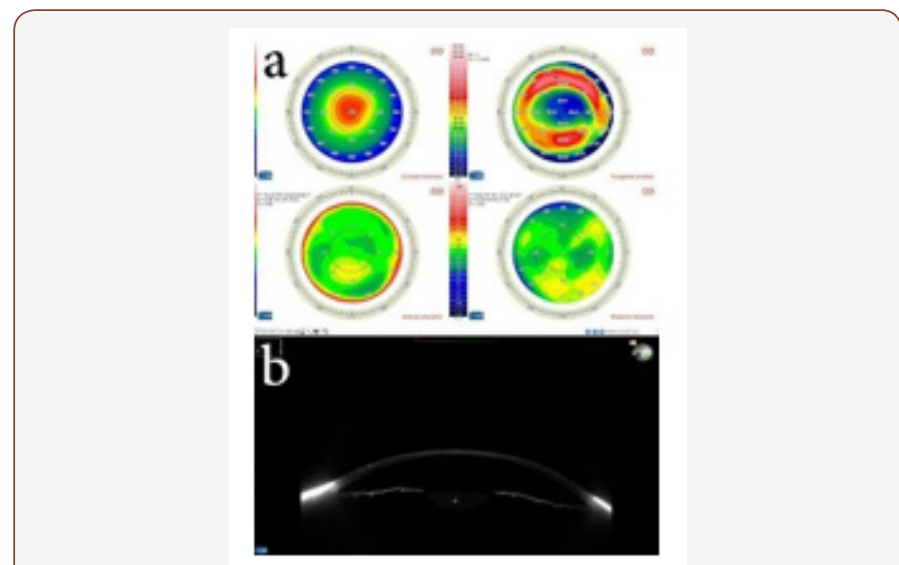

Figure 3: After 2 months a) Normal postlasik pentacam b) Scheimpflug imaging showed normal corneal contour.

\section{Discussion}

The technological progress of flap creation has emerged from mechanical manually guided microkeratomes to automated 
microkeratomes, single-use microkeratomes, and most recently to femtosecond (FS) laser technology [4, 5].

FS laser photo disrupts tissue at a preset depth and produces micro cavitation bubbles consisting of water and carbon dioxide. The bubbles expansion separates the corneal lamellae and flap creation [6]. Preliminary studies with the IntraLase FS laser have demonstrated lower rates of flap related complications in such as free flaps, irregular flaps, micro perforations, decentered flaps, epithelial defects, and abrasions [7-9].

In our case, although, the flap was created by femtosecond laser incomplete flaps had occurred due to repeated docking and femtosecond laser emission. In the first trial for flap creation by femtosecond laser, the flap wasn't centralized enough to allow sufficient ablation zone, so surgery was postponed and repeated again thereafter.

In a study of [10] they compare Laser in situ keratomileusis flap complications using mechanical microkeratome versus femtosecond laser, severe flap decentration had occurred in both groups with more liability in microkeratome group but with difference from our management that photorefractive keratectomy (PRK) was done for all cases of severe flap decentration.

Flap decentration in our case can be explained by minor movement of the patient's cornea with minimal tilting during femtosecond laser ablation leading to decentered created flap $[11,12]$. showed that flap adhesion strength in LASIK flaps made with a femtosecond laser was higher compared with a microkeratome due to good stromal wound healing to the extent that strong femto flap adhesion could be a disadvantage for patients of LASIK enhancement as this increase in flap edge healing interfere with re-lifting of the flap [13].

The second flap was created at the same depth of the first one (90um) because we expect complete flap healing as mentioned before especially no trial for decentered flap lifting had been done, also we were in need to create this thin flap to leave enough residual stromal bed adequate to treat as much as possible of the myopic error.

The second femtosecond laser ablation with the same depth created another flap encircling the previous decentered one and with the same bed.

We expected that decentered flap edge healing was complete; however, during flap lifting we found it did not heal completely leading to flap cleavage into two parts. In conclusion, flap decentration and incomplete flaps can occur with femtosecond laser flap creation. More notable conclusion is that femto flap edge healing is not so tight as thought before.

\section{Acknowledgment}

None.

\section{Conflicts of Interest}

The authors did not receive any financial support from any public or private sources. The authors have no financial or proprietary interest in a product, method, or material described herein.

\section{References}

1. Chen LY, Manche EE (2016) Comparison of femtosecond and excimer laser platforms available for corneal refractive surgery. Curr Opin Ophthalmol 27(4): 316-322.

2. Pietilä J, Huhtala A, Mäkinen P, Uusitalo H (2014) Flap characteristics, predictability, and safety of the Ziemer FEMTO LDV femtosecond laser with the disposable suction ring for LASIK. Eye (Lond) 28(1): 66-71.

3. Bashir ZS, Ali MH, Anwar A, Ayub MH, Butt N. (2017) Femto-lasik: The recent innovation in laser assisted refractive surgery. J Pak Med Assoc 67(4): 609-615.

4. Stonecipher K, Ignacio TS, Stonecipher M (2006) Advances in refractive surgery: microkeratome and femtosecond laser flap creation in relation to safety, efficacy, predictability, and biomechanical stability. Curr Opin Ophthalmol 17(4): 368-372.

5. Pietilä J (2012) Flap Creation in Laser-Assisted in Situ Keratomileusis: From Microkeratome to Femtosecond Laser. Tampere: Tampere University Press.

6. Kurtz RM, Liu X, Elner VM, Squier JA, Du D, et al. (1997) Photo disruption in the human cornea as a function of laser pulse width. J Refract Surg 13(7): 653-658

7. Kezirian GM, Stonecipher KG (2004) Comparison of the IntraLase femtosecond laser and mechanical keratomes for laser in situ keratomileusis. J Cataract Refract Surg 30(4): 804-811.

8. Binder PS (2004) Flap dimensions created with the IntraLase FS laser. J Cataract Refract Surg 30(1): 26-32.

9. Durrie DS, Kezirian GM (2005) Femtosecond laser versus mechanical keratome flaps in wave front-guided laser in situ keratomileusis: prospective contralateral eye study. J Cataract Refract Surg 31(1): 120126.

10. Moshirfar M, Gardiner JP, Schliesser JA, Espandar L, Feiz V, et al. (2010) Laser in situ keratomileusis flap complications using mechanical microkeratome versus femtosecond laser: retrospective comparison. J Cataract Refract Surg 36(11): 1925-33.

11. Kim JY, Kim MJ, Kim TI, Choi HJ, Pak JH et al. (2006) A femtosecond laser creates a stronger flap than a mechanical microkeratome. Invest Ophthalmol Vis Sci 47(2): 599-604.

12. Netto MV, Mohan RR, Medeiros FW, Dupps WJ, Sinha S et al. (2007) Femtosecond laser and microkeratome corneal flaps: comparison of stromal wound healing and inflammation. J Refract Surg 23(7): 667-676.

13. Santhiago MR, Smadja D, Zaleski K, Espana EM, Armstrong BK et al. (2012) Flap relift for retreatment after femtosecond laser-assisted LASIK. J Refract Surg 28(7): 482-487. 